\title{
Photoluminescence characterization of aged and regenerated mesoporous silica
}

\author{
C.M. Carbonaro ${ }^{\mathrm{a}, \mathrm{b}, *}$, P.C. Ricci ${ }^{\mathrm{a}}$, R. Corpino ${ }^{\mathrm{a}, \mathrm{b}}$, M. Marceddu $^{\mathrm{b}}$, A. Anedda $^{\mathrm{a}, \mathrm{b}}$ \\ a Dipartimento di Fisica, Università di Cagliari, s.p. n 8 Km 0.700, 09042 Monserrato, Cagliari, Italy \\ ${ }^{b}$ Centro Grandi Strumenti Università di Cagliari, s.p. n 8 Km 0.700, 09042 Monserrato, Cagliari, Italy
}

\section{A R T I C L E I N F O}

\section{Article history:}

Received 20 June 2010

Received in revised form 4 October 2010

Available online 28 December 2010

\section{Keywords:}

Porous silica;

Photoluminescence properties;

Synchrotron radiation

\begin{abstract}
A B S T R A C T
The investigation of the photoluminescence features of aged and regenerated mesoporous silica is reported. The emission spectrum of aged samples displays a blue band peaked at about $450 \mathrm{~nm}$ at room temperature with excitation channels at 250 and $200 \mathrm{~nm}$. No UV emission band is detected. The regenerated samples recover the optical transparency of the samples, the vibrational properties in the $3000-3800 \mathrm{~cm}^{-1}$ but for the isolated silanols band and the UV-blue emission features. Indeed beside the blue band peaked around $450 \mathrm{~nm}$ a UV band centred at $340 \mathrm{~nm}$ is reported whose relative contribution depends on the excitation wavelength. The aging and regeneration processes are discussed in view of the attribution of the observed emission features to specific surface defects.
\end{abstract}

(c) 2010 Elsevier B.V. All rights reserved.

\section{Introduction}

Visible light emission in silicon and silica-based nanostructured materials had gathered a lot of attention because of the possible technologic applications in the field of optoelectronics [1]. There are two main emission features: the red-mid IR S band and the blue-green $\mathrm{F}$ band. The first one is characterized by a decay time of several microseconds at room temperature and is related to the quantum confinement effect $[1,2]$. The second one shows a decay time in the nanosecond range and is related to a surface-defect model. The attribution of the $\mathrm{F}$ band to a defect is still debated since both carbonyl-related or hydroxyl-related surface defects have been proposed in the past [1-6]. It is well known indeed that the emission properties of nanostructured silica are affected both by thermal treatment, long term aging in air or water treatment [7-10]. Recently, a dehydroxylation reaction leading to the formation of a defect pair given by a dioxasilirane and a silylene center has been suggested as the origin of the observed blue emission [6]. It was also reported that the intensity and decay kinetics of the F band strongly depend on the porosity and microstructure of the starting material [7]. In addition to the $\mathrm{S}$ and $\mathrm{F}$ bands a composite UV band was reported and related, in a direct or indirect way, to the presence of silanol groups at the silica surface [11-16]. However also the attribution of the UV band is still questioned and C-based defects were proposed [17].

In this work we report the investigation of the emission properties of in air aged mesoporous silica as compared to regenerated samples via $\mathrm{O}_{2}$-rich water washing ant thermal treatment. Indeed the aging

\footnotetext{
* Corresponding author. Dipartimento di Fisica, Università di Cagliari, s.p. n $8 \mathrm{Km}$ 0.700, 09042 Monserrato, Cagliari, Italy. Tel.: + 39 0706754823; fax: + 39070510171. E-mail address: cm.carbonaro@dsf.unica.it (C.M. Carbonaro).
}

effects are well known in transparent porous glasses, for example in Vycor porous glass, and typically ascribed to the contamination by organic molecules adsorbed by the surrounding atmosphere $[18,19]$. The presence of contaminants modifies the transmittance properties of the material which turns from transparent to yellow or brown color depending on the nature and content of the organic adsorbed molecules. A possible solution to regenerate the porous glass is to wash the material with a strong oxidizer, such as $30 \%$ hydrogen peroxide, followed by heat treatment at about $100{ }^{\circ} \mathrm{C}$. The regeneration process is successfully accomplished when the material turns back to transparency. The purposes of the work are two: from one side to investigate the spectroscopic features of the aged silica and to verify the extent of the regeneration process; from the other side the adsorption of organic molecules and the cleaning procedure offer the opportunity to shed some light on the attribution of the UV and blue-green bands.

\section{Samples and experiments}

The investigated samples are sol-gel synthesized commercial porous silica monoliths (diameter $5 \mathrm{~mm}$, thickness $2 \mathrm{~mm}$ ) produced by Geltech Inc. (US) with pore diameter distribution sharply peaked at $7.5 \mathrm{~nm}$ (BET analysis, $5 \%$ of standard deviation), a pore volume of $1.000 \mathrm{~cm}^{3} \mathrm{~g}^{-1}$ with a specific surface area of $525 \mathrm{~m}^{2} \mathrm{~g}^{-1}$ and a density of about $0.7 \mathrm{~g} \mathrm{~cm}^{-3}$. The aged samples were kept in ambient atmosphere for a few years and show a diminished transparency in the visible range because of a slightly brown homogeneous color typically due to moisture adsorption. Treated samples were washed with $\mathrm{O}_{2}$-rich water (30\% hydrogenated peroxide) and heated at $90{ }^{\circ} \mathrm{C}$ until the transparency was restored (wet annealing) [18].

Photoluminescence (PL) and excitation of PL (PLE) measurements were carried out at the SUPERLUMI experimental station on the I 
beamline of the HASYLAB in Desy (Hamburg) by using the pulsed excitation light of the synchrotron radiation (SR). The PLE measurements were performed in the $4-10 \mathrm{eV}$ energy range $(0.3 \mathrm{~nm}$ of bandwidth) and corrected for the spectral efficiency of the excitation source. The PL signal was dispersed by a Spectra-Pro 300i monochromator and detected in the 1.5-5.0 eV energy range with a LN-CCD (Princeton Instruments) for the PL measurements, and with a photomultiplier (Hamamatsu 206358P) for the PLE measurements. PL and PLE spectra were recorded under multi-bunch operation and detected with an integral time window of 192 ns correlated to the SR pulses. Decay time in the ns domain was gathered under a singlebunch operation, using 1024 channels to scan the 192 ns interval time between adjacent pulses (pulse width of $0.2 \mathrm{~ns}$ ). A continuous-flow liquid helium cryostat was used to vary the temperature of the sample chamber in the 300-8 K range.

\section{Results}

The regeneration procedure allows to restore the optical transparency of the samples and the pristine optical properties. Indeed, the Raman spectrum of the regenerated sample in the $2800-3800 \mathrm{~cm}^{-1}$ range displayed the contribution of different hydrogen-bonding interacting hydroxyl species, as reported for the raw material [15], but no sign of isolated silanols. On the contrary, the Raman spectrum of the aged samples in the same spectral range could not be detected because of the overlapping of a large visible luminescence band. In Fig. 1 the room temperature emission spectra of the two samples recorded under $250 \mathrm{~nm}$ excitation are reported. The regenerated samples show the contribution of two bands, in the UV and visible range respectively, in agreement with the previously published findings but with a smaller contribution of the UV band [20,21]. The aged samples show a very large contribution of a band in the visible range and no emission in the UV range. The visible band is structured in both samples: the emission peaks are positioned at about 430 and $460 \mathrm{~nm}$ with different contributions in the two samples. When the emission spectra are recorded at low temperature $(8 \mathrm{~K})$ the PL peaks are still resolved in the regenerated samples and the contribution of the UV band is larger (Fig. 2). The aged samples display a smooth broad visible band peaked at $460 \mathrm{~nm}$ and still no contribution in the UV range. The relative amplitude of the visible band with respect to the UV one in regenerated samples depends on the excitation

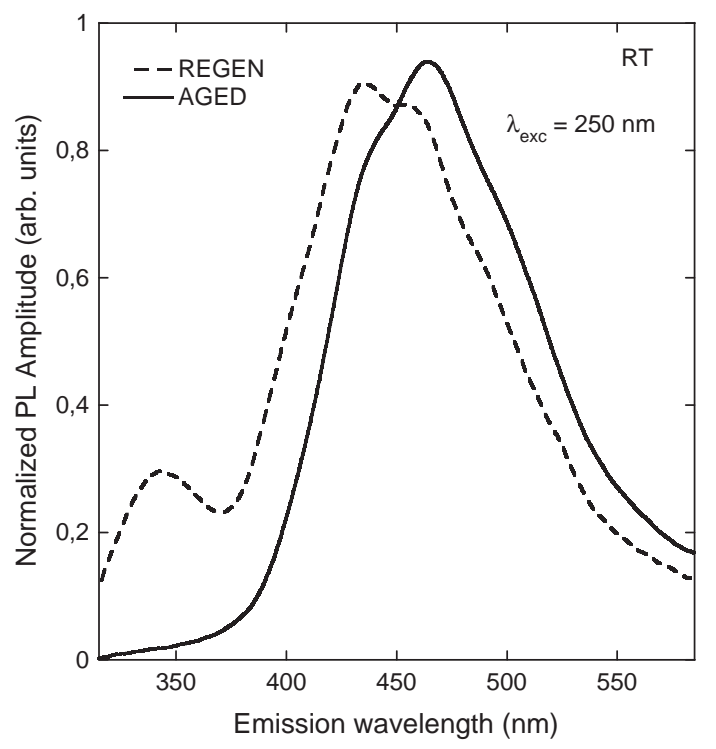

Fig. 1. PL excited at $250 \mathrm{~nm}$ at room temperature.

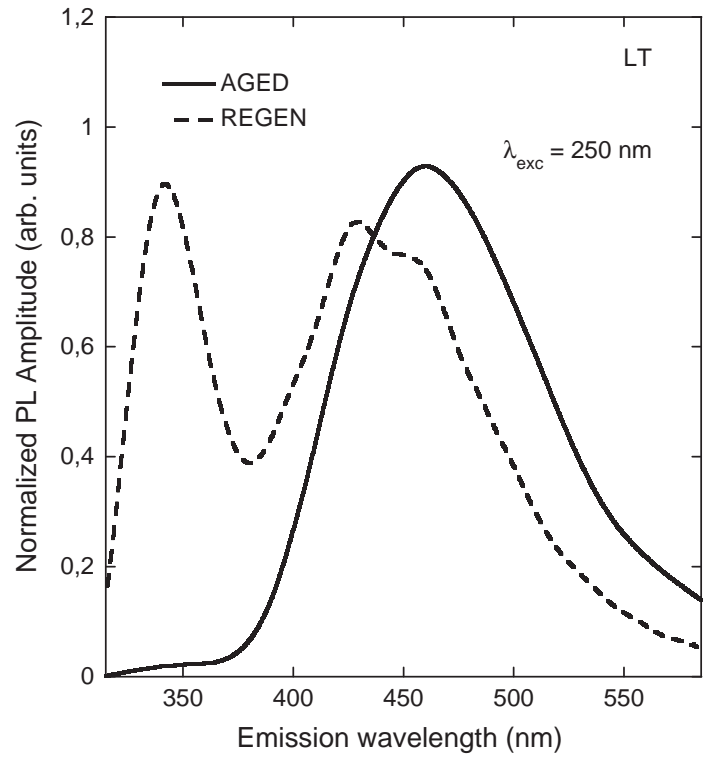

Fig. 2. PL excited at $250 \mathrm{~nm}$ at $8 \mathrm{~K}$.

wavelength, showing that, at room temperature, it decreases as the excitation wavelength decreases (Fig. 3a).

The aged samples display a visible band that blue shifts as the excitation wavelength decreases because of the different relative contribution of the resolved peaks and a contribution in the UV range is observed by exciting at $175 \mathrm{~nm}$ (Fig. 3b).

The PLE spectra of the blue band are reported in Fig. 4: The spectra were recorded by monitoring the emission peak at $445 \mathrm{~nm}$ at room temperature (RT) and the emission peak at $430 \mathrm{~nm}$ at low temperature $(8 \mathrm{~K})$. The two set of samples show the same PLE spectrum at RT

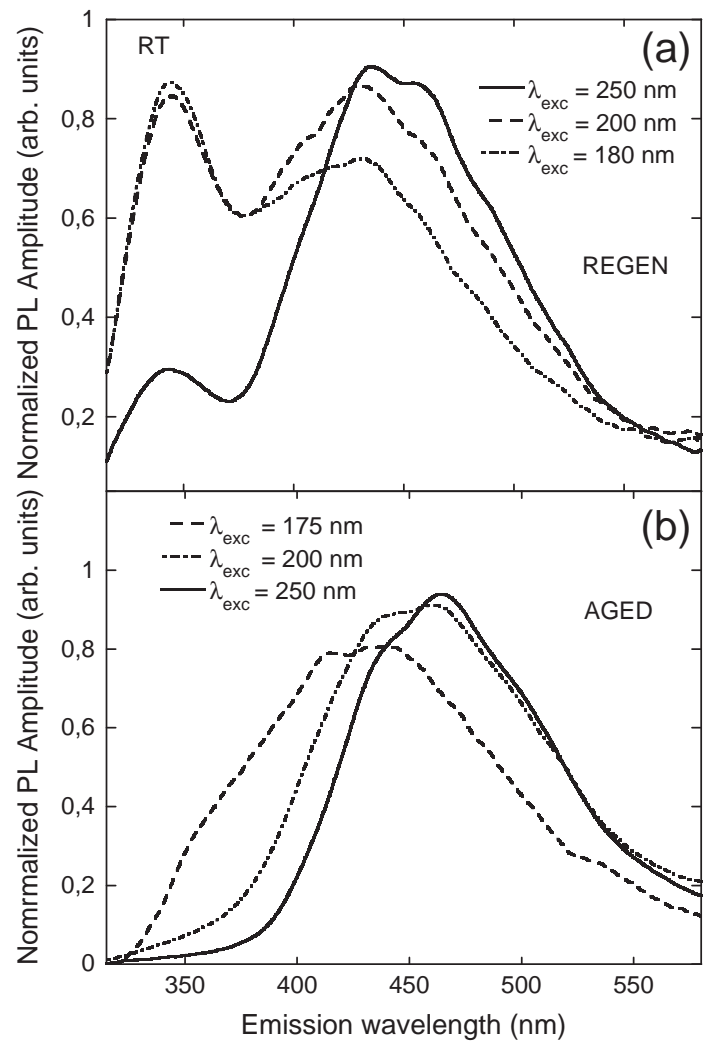

Fig. 3. PL spectra recorded at different excitation wavelengths at room temperature. 


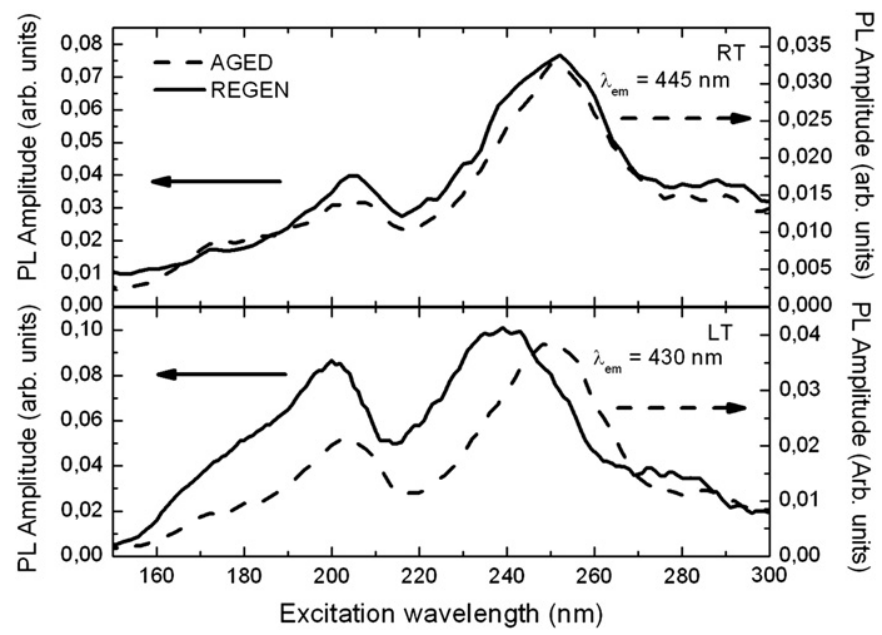

Fig. 4. PLE spectra recorded at different emission wavelengths at room temperature (RT) and $8 \mathrm{~K}(\mathrm{LT})$.

with two main excitation channels at 250 and $200 \mathrm{~nm}$. When the spectra are recorded at $8 \mathrm{~K}$ a blue shift of the excitation peak from 250 to $240 \mathrm{~nm}$ is observed in the regenerated samples coupled with the increase of a shoulder at about $175 \mathrm{~nm}$. On the contrary the low temperature PLE spectrum of the aged samples is comparable with the one recorded at RT.

The UV excited decay time of samples (emission wavelength $430 \mathrm{~nm}$ ) recorded at $8 \mathrm{~K}$ are reported in the inset of Fig. 5 . The time profiles call for a non-single exponential decay law: indeed the experimental data can be successfully fitted with two exponential decays and two different lifetimes, of about 2 ns and above 20 ns, can be extracted for the regenerated samples. In the case of the aged ones the second lifetime is slightly shorter, of about $13 \mathrm{~ns}$. The data analysis

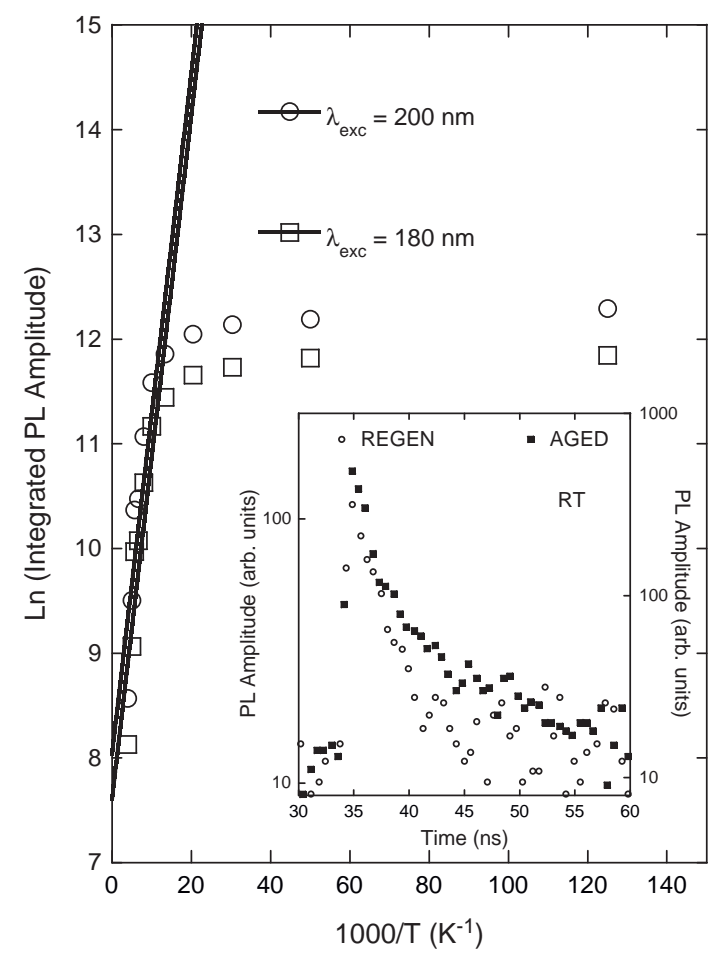

Fig. 5. Arrhenius plot of the integrated amplitude of the blue band excited at 180 and $200 \mathrm{~nm}$ for the aged samples. The inset reports the decay time of the regenerated and aged samples excited at $180 \mathrm{~nm}$ and recorded at $430 \mathrm{~nm}$ at RT. was performed at different excitation wavelengths (175, 200 and $250 \mathrm{~nm}$, both at RT and LT) and recording the signal at different emission wavelength of the blue band, namely 430, 445 and $460 \mathrm{~nm}$ : the general scenario is the one described before, two exponential decays being always needed to achieve a good approximation of the experimental data. As previously reported $[20,21]$ the decay time profiles indicate the presence of two emission components with fast ( $2 \mathrm{~ns}$ ) and slow (above $20 \mathrm{~ns}$ but shorter for the aged samples) lifetimes. It should be noted, however, that the small signal to noise ratio does not allow a more precise determination of the investigated lifetimes and further time resolved experiments are planned to better clarify the time kinetic of the blue band.

The temperature dependence of the blue band recorded in aged samples is reported in Fig. 5 through the Arrhenius plot of the integrated PL amplitude excited both at 200 and $180 \mathrm{~nm}$. In both cases an activation energy of about $30 \pm 4 \mathrm{meV}$ can be calculated (linear correlation factor $\mathrm{R}>0.90$ ).

\section{Discussion}

The aging process causes modifications of the spectroscopic features of mesoporous silica which are visible at naked eye. Indeed the transmittance is largely reduced and the material turns to yellow or brown color because of the adsorption of organic molecules from the surrounding atmosphere. Since mesoporous silica have been largely investigated in the recent past because of their emissions in the UV and visible range for possible technological applications and because of their relationship to the emission properties of silicon based nanostructure $[1,2,22]$, the investigation of the effects of the aging process on the emission properties can help to clarify the nature and attribution of the emissions reported so far. In order to recover the pristine transparency property a regeneration procedure can be applied where the contaminated samples are treated with a strong oxidizer, such as $30 \%$ hydrogen peroxide, and heated at about $90-$ $100{ }^{\circ} \mathrm{C}$ to allow the elimination of the residual contaminants. The analysis of the Raman spectrum in the $3000-3800 \mathrm{~cm}^{-1}$ range allows to assess that the regeneration procedure restored the pristine properties of the material. Indeed the observed spectrum is comparable to previously reported ones [15] but for the lack of isolated silanols because of the large amount of water engulfed in the porous matrix. On the contrary the presence of a large visible band overlapping the vibrational spectrum of the aged samples is a further proof of the presence of contaminants at the silica surface. The analysis of the emission properties of aged and regenerated samples indicated the contribution of emission bands in the UV-visible (blue) range. The attribution of the UV and blue emission bands reported in the literature was largely discussed: it is now accepted that both the emissions are related, in a direct or indirect way, to the presence of $\mathrm{OH}$ groups at the surface of the porous matrix. In particular it was reported that the blue emission band depends on the microscopic morphology and the porosity of the samples [7]. In addition the PL amplitude increases by annealing the samples in the $200-400{ }^{\circ} \mathrm{C}$ and is largely quenched by annealing the samples above $600{ }^{\circ} \mathrm{C}$ [7]. A recent model proposed to explain these spectroscopic features is based on the dehydroxylation reaction of two geminal silanols at the silica surface to produce a defect pair consisting of a dioxasilirane and a silylene center [6]. Concerning the UV band the relationship to the relative content of silanol species present at the silica surface was proposed [11-16]. It must be stressed, however, that the contribution of C-related centers was claimed as a possible explanation of the reported emissions [17]. Indeed by exposing the porous matrix to ambient air both organic molecules and $\mathrm{OH}$ groups can be adsorbed at the silica surface, so that the aging process can be exploited to elucidate the attribution of the emission bands. In addition the regeneration procedure, being performed with $\mathrm{O}_{2}$-rich water, can also help to clarify these attributions. 
The main result we can deduce from the comparison of the emission features of aged and regenerated samples is that, despite small differences related to the morphology of the samples (the regeneration procedure is expected to modify the microstructure of the material), the observed blue emission is the same in both the samples. On the contrary, while the regeneration procedure restores the emission properties of the samples in the UV and visible range, the aged samples do not display any contribution in the UV range but for a small shoulder when excited at $175 \mathrm{~nm}$. If the UV emission band was related to the presence of carbonyl species, we should have detected the increase of this band in the aged samples: since the long term aging process quenches the relative contribution of the UV band, the latter could not be ascribed to the presence of C-based surface defects. On the contrary, according to the hypothesis of OH-related UV band, the presence of adsorbed organic species at the silica surface is expected to react with the isolated silanols to produce hydrogen bonded C-related defects [17], thus reducing the relative contribution of the UV band.

It was also reported that the blue emission is not related to contaminant species, since it was observed in high-purity nanostructured and mesoporous silica [6]. Since the adsorption on the silica surface of $\mathrm{OH}$ groups is accomplished both during the aging process and the regeneration procedure, both the processes can favor the formation of the visible band because of the formation of geminal silanols required for the dehydroxylation reaction: the reaction among adjacent geminal silanols is thermodynamically driven during the aging process (the adsorption and desorption of molecules is a dynamic process at the porous surface) $[23,24]$ or is accomplished because of the wet annealing around $100{ }^{\circ} \mathrm{C}$ during the regeneration procedure.

The regeneration procedure can be regarded as a sort of chemically controlled aging process, so that a modification of the morphology and the porosity of the samples is expected in both cases [23,24]. The main difference is in the relative content of molecular species expected to be trapped at the porous matrix: regenerated samples contain $\mathrm{OH}$ species and engulfed $\mathrm{H}_{2} \mathrm{O}$, aged samples contain also organic molecules. The expected morphology changes allow to justify the small spectroscopic differences recorded for the blue emission in aged and regenerated samples. Four different processes can occur during the investigated treatments: polycondensation, syneresis, coarsening, and phase transformation [23,24]. The modifications of the silica microstructure depend on different parameters, such as time, temperature, and $\mathrm{pH}$, and different effects can be observed: an increase of the network connectivity (polycondensation), a spontaneous shrinkage of the matrix (syneresis), and a variation in the mean pore diameter with a change of the specific surface because of dissolution and reprecipitation (coarsening). As a consequence, the emitting centers sense different local environments because of the modification of the chemical surroundings induced by the aging or the regeneration. It was reported, for example, that a bimodal distribution of the pore diameter can be observed in $\mathrm{SiO}_{2}$ xerogel samples: the regeneration procedure, and, to somehow extent, the aging process, increases the diameter of the micropores (diameter $<2 \mathrm{~nm}$ ) and decreases the one of the mesopores $(2<$ diameter $<50 \mathrm{~nm})$ [25]. The change in the pore diameter distribution affects the observed spectroscopic features because of the modification of the distribution of different $\mathrm{OH}$ groups. In this regard we can also understand the differences observed among regenerated and as received samples $[20,21]$, in particular the smaller relative contribution of the UV band in the former ones: the emission recorded at $8 \mathrm{~K}$ in regenerated samples (Fig. 3) is comparable to the one observed in as received samples with larger pore diameter under the same experimental conditions.
A final comment pertains to the measured activation energy: the value estimated for the as received samples is of about $40 \mathrm{meV}$, in the regenerated samples the estimated value is around $30 \mathrm{meV}$ [16]. The small difference can be ascribed to the different microstructure and pore distribution of the set of samples and explains the quite small signal detected at room temperature.

\section{Conclusions}

In the present paper we investigated, from a spectroscopic point of view, the aging effects on mesoporous silica and the possibility to recover the optical features of the aged samples, in terms of UV and visible emissions, through a regeneration procedure. The reported results also help to clarify the attribution of the observed emissions to specific surface centers. It was shown that, due to the aging process, the UV emission disappears while the blue emission displays spectroscopic features comparable to that of the as received samples. The regeneration process allows to restore both the UV and visible bands but the relative contribution of the two emissions depends on the modification induced on the microscopic morphology by the process itself. The reported data indicate that the UV emission cannot be related to the presence of organic contaminants at the silica surface and that the blue band of both aged and regenerated samples can be explained by a recently proposed dehydroxylation reaction model.

\section{Acknowledgment}

The research leading to these results has received funding from the European Community's Seventh Framework Programme (FP7/20072013) under grant agreement no. 226716.

\section{References}

[1] A.G. Cullis, L.T. Canham, P.D. Calcot, J. Appl. Phys. 82 (1997) 909

[2] A.G. Cullis, L.T. Canham, Nat. Lond. 353 (1991) 335.

[3] H. Koyama, N. Koshida, Solid State Commun. 103 (1997) 37.

[4] A.J. Kontkiewicz, A.M. Kontkiewicz, J. Siejka, S. Sen, G. Novak, A.M. Hoff, P. Sakthivel, K. Ahmed, P. Mukherjee, S. Witanachchi, J. Lagowski, Appl. Phys. Lett. 65 (1994) 1346.

[5] N. Sagawa, T. Uchino, Appl. Phys. Lett. 87 (2005) 251923.

[6] T. Uchino, N. Kurumoto, N. Sagawa, Phys. Rev. B 73 (2006) 233203.

[7] Y. Nakazaki, K. Fujita, K. Tanaka, T. Uchino, J. Phys. Chem. C 112 (2008) 10878.

[8] H.J. Chang, Y.F. Chen, H.P. Lin, C.Y. Mou, Appl. Phys. Lett. 78 (2001) 3791.

[9] M. Zhang, E. Ciocan, Y. Bando, K. Wada, L.L. Cheng, P. Pirouz, Appl. Phys. Lett. 80 (2002) 491.

[10] H. Koyama, Y. Matsushita, N. Koshida, J. Appl. Phys. 83 (1998) 1776

[11] B. Yao, H. Shi, X. Zhang, L. Shang, Appl. Phys. Lett. 78 (2001) 174

[12] A. Anedda, C.M. Carbonaro, F. Clemente, R. Corpino, P.C. Ricci, J. Phys. Chem. B 109 (2005) 1239

[13] C.M. Carbonaro, F. Clemente, R. Corpino, P.C. Ricci, A. Anedda, J. Phys. Chem. B 109 (2005) 14441.

[14] A. Anedda, C.M. Carbonaro, F. Clemente, R. Corpino, S. Grandi, P. Mustarelli, A. Magistris, J. NonCryst. Solids 322 (2003) 68

[15] A. Anedda, C.M. Carbonaro, F. Clemente, R. Corpino, P.C. Ricci, J. Phys. Chem. B 107 (2003) 13661.

[16] C.M. Carbonaro, P.C. Ricci, A. Anedda, Phys. Rev. B 76 (2007) 125431

[17] H. He, Y. Wang, H. Tang, J. Phys. Condens. Matter 14 (2002) 11867.

[18] Vycor brand porous glass 7930 datasheet by Corning, http://www.corning.com.

[19] S. Stapf, Nat. Phys. 2 (2006) 731-732.

[20] A. Anedda, C.M. Carbonaro, F. Clemente, R. Corpino, P.C. Ricci, Opt. Mat. 27 (2005) 958.

[21] A. Anedda, C.M. Carbonaro, F. Clemente, R. Corpino, P.C. Ricci, Mater. Sci. Eng. C 25 (2005) 631

[22] G.G. Qin, J. Lin, J.Q. Duan, G.Q. Yao, Appl. Phys. Lett. 69 (1996) 1689.

[23] C.J. Brinker, G.W. Schere, Sol Gel Science: The Physics and Chemistry of Sol-Gel Processing, Academic Press, San Diego, 1990.

[24] L.L. Hench, J.K. West, Chem. Rev. 90 (1990) 33.

[25] S. Grandi, P. Mustarelli, C. Tomasi, G. Sorarù, G. Spanò, J. NonCryst. Solids 343 (2004) 71. 